\title{
CONDUTIVIDADE HIDRÁULICA NÃO SATURADA DE UM PODZÓLICO AMARELO DA ZONA DA MATA NORTE DE PERNAMBUCO ${ }^{1}$
}

\author{
ANDRÉ MACIEL NETTO ${ }^{2}$, ANTONIO CELSO DANTAS ANTONINO ${ }^{3}$, PIERREAUDRY ${ }^{4}$, \\ CLEMENTE JOSÉ GUSMAO CARNEIRO5 e ATTÍLIO DALL'OLIO ${ }^{6}$
}

\begin{abstract}
RESUMO - A determinação da condutividade hidráulica de um Podzólico Amarelo foi obtida a partir de experimento montado em uma parcela de 3,5 m x 3,5 m, localizada na Estação Experimental da Empresa Pernambucana de Pesquisa Agropecuária (IPA), de Itapirema, Goiana, PE. Foi utilizado o método de drenagem interna para calcular a condutividade hidráulica $(\mathrm{K}(\theta))$ como função da umidade do solo, nos três horizontes característicos do solo. Os perfis de umidade e de potencial matricial, em função do tempo, obtidos no experimento de drenagem interna mostram claramente a existência de um joelho que marca a transição entre as fases de drenagem rápida e lenta. Várias funções do tipo potência, soma de duas e três exponenciais, foram usadas para modelar essa transição entre a fase rápida e a fase lenta de drenagem. O horizonte $\mathrm{A}$, arenoso, com poros maiores, é um horizonte de elevada condutividade hidráulica, enquanto o horizonte BA, de estrutura maciça com poros pouco visíveis, é um horizonte de impedimento para a infiltração. A dinâmica hídrica do horizonte Bt é mais complexa em virtude de sua heterogeneidade. A caracterização dos horizontes A e BA pode trazer subsídios significativos para a elaboração de modelos de simulação numérica dos processos de transferência de água nos horizontes superficiais, que são os mais condicionantes para a exploração agrícola e a conservação dos solos.
\end{abstract}

Termos para indexação: propriedades hidráulicas do solo, água do solo, permeabilidade, drenagem, infiltração.

\section{UNSATURATED HYDRAULIC CONDUCTIVITY OF A YELLOW PODZOLIC SOIL} IN THE NORTH FOREST ZONE OF PERNAMBUCO STATE

\begin{abstract}
The determination of the hydraulic conductivity of a Yellow Podzolic soil was carried out during an experiment in a plot measuring $3.5 \mathrm{~m}$ x $3.5 \mathrm{~m}$, at the Estação Experimental de Itapirema, Goiana, in the State of Pernambuco, Brazil. The internal drainage method was used to obtain the hydraulic conductivity $(\mathrm{K}(\theta))$ as a function of soil water content, in the three main horizons of the soil. In relation to the methodological aspects, processing of data from internal drainage experiments, including the initial phase of fast drainage, the adjustment of the required parameters, it is necessary to use functions that reproduce the distinct transition between the fast and slow phases of drainage. From all five tested functions, those of power, sum of two exponentials and sum of three exponentials, especially this last one, adjusted well to this distinct transition. Three main horizons of the Yellow Podzolic soil were investigated for hydraulic conductivity. The sandy A horizon, with large pores, has a high conductivity while the BA horizon, with massive structure and few visible pores, has a low infiltration rate. The hydraulic dynamics of the Bt horizon is more complex due to its heterogeneity. The precise characterization of the A and BA horizons, which are the most important to agriculture and soil conservation, makes it possible to elaborate numeric simulation models of the water transference process in the surface horizons of this type of soil.
\end{abstract}

Index terms: soil hydraulic properties, soil water, permeability, drainage, infiltration.

\footnotetext{
${ }^{1}$ Aceito para publicação em 14 de janeiro de 2000.

${ }^{2}$ Físico, Ph.D., Universidade Federal de Pernambuco (UFPE), Departamento de Energia Nuclear-Centro de Tecnologia, CEP 50540-740 Recife, PE. E-mail: amnetto@npd.ufpe.br

${ }^{3}$ Eng. Civil, Ph.D., Prof. Adjunto, UFPE. E-mail: 02ACDA@npd.ufpe.br
}

\footnotetext{
${ }^{4}$ Pedólogo, Institut Français de Recherche Scientifique pour le Développement en Coopération (ORSTOM)/UFPE E-mail: pierreaud@aol.com.br

${ }^{5}$ Eng. Elétr., Ph.D., UFPE. E-mail: clemente@npd.ufpe.br

${ }^{6}$ Físico, M.Sc., Prof. Adjunto, UFPE. E-mail: ado@elogica.com.br
} 


\section{INTRODUÇÃO}

O movimento da água no solo é um processo essencialmente contínuo. Contudo, para fins de análise, são identificadas várias fases e estados desse movimento, a saber: a infiltração no solo, o armazenamento, a drenagem, a evaporação e a absorção pelas plantas. O movimento da água controla a dinâmica dos elementos químicos que intervêm nos processos de formação e evolução dos solos, na disponibilidade de nutrientes para as plantas e na satisfação da demanda hídrica. Por sua vez, esse movimento depende das características físico-químicas do solo, quais sejam, textura, estrutura e teor de matéria orgânica.

$\mathrm{Na}$ Zona da Mata Norte de Pernambuco ocorrem grandes áreas de Podzólicos Amarelos que têm seu potencial quase totalmente explorado pela monocultura da cana-de-açúcar. Além dos problemas relacionados com a baixa fertilidade química, a disponibilidade de água constitui um dos fatores limitantes para a otimização da produção agrícola nesses solos, tornando-se necessário o conhecimento de suas propriedades hidrodinâmicas.

Hillel et al. (1972) descreveram detalhadamente um procedimento simplificado para medir a condutividade hidráulica do solo $(K(\theta))$, baseado na monitoração do estado transiente da drenagem interna de um perfil de solo. Ele permite determinar as propriedades hidráulicas desse perfil a partir de medidas freqüentes e simultâneas da umidade e potencial matricial, através de sonda de neutrons e de tensiômetros com manômetro de mercúrio, em várias profundidades.

A interpretação dos dados coletados em experiências de drenagem interna sempre necessita de ajustes da umidade $(\theta)$ e o armazenamento de água $(W)$, medidos de maneira descontínua ao longo do tempo $(t)$, obtendo-se curvas contínuas $\theta(\mathrm{t})$ e $\mathrm{W}(\mathrm{t})$, a fim de permitir interpolações e derivações para o cálculo dos fluxos.

O método manual de ajuste pode ser considerado como referência, pois não comporta nenhuma hipótese a priori sobre a forma das curvas. Entretanto, ele não é reprodutível e pode incluir um certo grau de subjetividade, além de ser muito trabalhoso. Por isso, numerosas tentativas de ajustes analíticos encontram-se na literatura.
Hillel et al. (1972) ajustaram com sucesso a evolução da umidade a uma equação do tipo $\theta=a+b \cdot \log (t)$. Esta forma logarítmica foi bastante utilizada, com várias adaptações (Saunders, 1978; Libardi et al., 1980). Chong et al. (1981) utilizaram uma função potência para descrever a evolução da umidade com o tempo; esta forma foi também bastante utilizada por Prevedello et al. (1981) e Vachaud et al. (1991).

O objetivo deste trabalho foi determinar a condutividade hidráulica, em regime de drenagem interna, de um Podzólico Amarelo, dando particular ênfase às metodologias de ajuste de funções aos dados experimentais.

\section{MATERIAL E MÉTODOS}

O experimento foi conduzido na área experimental da Empresa Pernambucana de Pesquisa Agropecuária (IPA), em Itapirema, Goiana, Zona da Mata Norte do Estado de Pernambuco, (latitude $7^{\circ} 37^{\prime} 30^{\prime \prime} \mathrm{S}$, longitude $34^{\circ} 57^{\prime} 30^{\prime \prime O}$ e altitude de $85 \mathrm{~m}$ ). A precipitação pluvial total e a temperatura média anuais em Itapirema são da ordem de $2.000 \mathrm{~mm} \mathrm{e} 25^{\circ} \mathrm{C}$, respectivamente.

Os solos da região de Itapirema derivam do grupo Barreiras, constituídos de sedimentos de origem continental do final do terciário, de textura arenosa até argilosa, caracterizados por uma alteração intensa (Brasil, 1969, 1972). A área escolhida para o estudo está localizada na parte alta da estação experimental e apresenta solos Podzólicos Amarelos latossólicos, com drenagem moderada. A descrição do perfil do solo estudado pode ser resumida da maneira a seguir: 1) de 0 a $45 \mathrm{~cm}$ : conjunto de horizontes $\mathrm{A}+\mathrm{AB}$, de textura leve, com um teor de argila menor que $10 \%$ na parte superficial, aumentando até cerca de $15 \%$ na parte inferior. Esse conjunto, com muitos poros grandes e presença de raízes abundantes, proporciona uma alta permeabilidade; 2) de 45 a $80 \mathrm{~cm}$ : um horizonte BA, franco-argilo-arenoso ( $25 \%$ a $30 \%$ de argila), coeso e de estrutura maciça, com poucos poros visíveis, duro quando seco e firme quando úmido. Esse horizonte, típico dos Podzólicos Amarelos, é considerado como nível de impedimento tanto para o enraizamento das culturas como para infiltração e drenagem; 3) acima de $80 \mathrm{~cm}$ : um horizonte Bt, de textura franco-argilo-arenosa, aparentemente mais permeável e menos denso que o horizonte BA sobrejacente, por possuir uma estrutura microagregada do tipo que se observa em material latossólico, mesmo que moderadamente desenvolvida. 
Para a determinação da $\mathrm{K}(\theta)$ em função da umidade, nos três horizontes, utilizou-se o procedimento de Hillel et al. (1972). Foi obtida através de:

$K(\theta)=\frac{[\mathrm{dW} / \mathrm{dt}]_{2}}{[\partial \psi / \partial \mathrm{z}]_{2}}$

onde:

W representa o estoque acumulado de água (L), $\Psi$ é o potencial total da água no solo (L), z é a profundidade do perfil (L), positiva para baixo e t o tempo (T).

A densidade de fluxo foi obtida utilizando-se, para diferentes tempos, o cálculo do estoque acumulado de água compreendido entre a superfície do solo e o nível de interesse $\mathrm{z}$, integrando-se os perfís de umidade e traçando-se a curva $\mathrm{W}(\mathrm{z}, \mathrm{t})$

Para o experimento 1 de drenagem interna, delimitouse uma parcela de $3,5 \mathrm{~m} \times 3,5 \mathrm{~m}$. Nos quatro lados da parcela foram abertas trincheiras, até a profundidade de 1,6 m isolando-se, assim, um bloco de solo. As paredes do bloco foram cobertas com plástico preto para assegurar a verticalidade do fluxo, conforme exigência do método. Após a colocação do plástico, as trincheiras foram fechadas, recolocando-se o solo adequadamente compactado. Nessa parcela foi instalado um tubo de alumínio de $2,70 \mathrm{~m}$ de profundidade para o acesso da sonda de neutrons. Ao redor do tubo foram instalados, a uma distância de $30 \mathrm{~cm}$ do eixo do tubo, oito tensiômetros, nas profundidades de 10 , $20,30,40,50,70,90,110$ e $130 \mathrm{~cm}$

O experimento 2 de drenagem interna foi realizado após retirar os primeiros $80 \mathrm{~cm}$ de solo, com o objetivo de se obter, para os horizontes profundos, uma maior amplitude nos valores de umidade e, portanto, melhores condições para a determinação da condutividade hidráulica.

A parcela foi inundada até que as leituras dos tensiômetros não mais variassem, atingindo-se uma quase saturação do perfil em estudo, o que se verificou depois de quatro horas do início da inundação. A seguir, retirou-se o excesso de água sobre a superfície do solo, que foi coberta com plástico, para impedir qualquer fluxo de água (evaporação ou infiltração). Sobre o plástico, colocou-se uma camada de solo para segurá-lo e para evitar excessivo aquecimento. Para proteger a parcela da chuva, foi montada uma tenda sobre ela. Os estudos de drenagem interna foram realizados em 36 e 41 dias com os experimentos 1 e 2 , respectivamente.

Durante as fases de drenagem interna da água, foram realizadas medidas de perfis neutrônicos de $15 \mathrm{em} 15 \mathrm{~cm}$ até a profundidade de $150 \mathrm{~cm}$ e, a partir desta, de $30 \mathrm{em}$ $30 \mathrm{~cm}$ até a profundidade de $270 \mathrm{~cm}$. Na fase inicial, de evolução mais rápida, realizaram-se medidas freqüentes dos perfis neutrônicos e de tensiometria a intervalos de $2 \mathrm{e}$
5 minutos na primeira hora de ensaio; de 15 e 30 minutos na segunda hora, prosseguindo-se com intervalos de 1 hora no segundo dia. No terceiro dia, foram efetuadas três leituras diárias, seguindo-se com duas leituras diárias após o quarto dia, sendo uma pela manhã e outra à tarde

Para as medidas de umidade, foi utilizada uma sonda de nêutrons TROXLER série 3300, que contém uma fonte radioativa de $\mathrm{Am}-\mathrm{Be}$ de atividade de $3,7.10^{8} \mathrm{~Bq}(10 \mathrm{mCi})$. Durante as medidas foi utilizado um disco refletor com $20 \mathrm{~cm}$ de diâmetro para minimizar a fuga de nêutrons pela superfície do solo.

Dois métodos, manual e analítico, foram usados para ajustar os dados experimentais das evoluções de umidade, de potencial total da água no solo e de estoque acumulado de água dos dois ensaios de drenagem interna. No ajuste manual, as curvas foram cuidadosamente delineadas utilizando-se curvas francesas, enquanto a densidade de fluxo foi obtida a partir da curva de estoque ajustada, empregando-se o método do espelho. No caso dos ajustes analíticos, foram ajustadas várias funções pelo método Quasi-Newton, utilizando-se como função perda a soma dos quadrados dos desvios (Tabela 1).

\section{RESULTADOS E DISCUSSÃO}

Os coeficientes lineares e angulares das retas de calibração para a sonda de neutrons utilizada foram obtidos a partir dos componentes da análise da variância (Tabela 2).

A evolução do potencial total da água no solo e as respectivas curvas ajustadas por uma tripla exponencial, para os experimentos 1 e 2, em diferentes profundidades, estão mostrados na Fig. 1. Em

TABELA 1. Funções utilizadas nos ajustes analíticos.

\begin{tabular}{ll}
\hline Função & Equação $^{1}$ \\
\hline Logarítmica & $\mathrm{Y}=\mathrm{A}+\mathrm{B} \cdot \log (\mathrm{x})$ \\
Potência & $\mathrm{Y}=\mathrm{Ax}+\mathrm{Bx} \mathrm{C}^{\mathrm{C}}+\mathrm{D}$ \\
Duas potências & $\mathrm{Y}=\mathrm{Ax}+\mathrm{Bx}^{\mathrm{C}}+\mathrm{Dx} \mathrm{E}^{\mathrm{E}}+\mathrm{F}$ \\
Duas exponenciais & $\mathrm{Y}=\mathrm{A}\left(\mathrm{e}^{-(\mathrm{Bx})}-1\right)+\mathrm{C}\left(\mathrm{e}^{-(\mathrm{Dx})}-1\right)+\mathrm{E}$ \\
Três exponenciais & $\mathrm{Y}=\mathrm{A}\left(\mathrm{e}^{-(\mathrm{Bx})}-1\right)+\mathrm{C}\left(\mathrm{e}^{-(\mathrm{Dx})}-1\right)+\mathrm{E}\left(\mathrm{e}^{-(\mathrm{Fx})}-1\right)+\mathrm{G}$ \\
\hline
\end{tabular}

${ }^{1}$ Y: representa os parâmetros hídricos (umidade, estoque de água e potencial total); $\mathrm{x}$ : tempo

TABELA 2. Parâmetros das retas de calibração neutrônica.

\begin{tabular}{ccc}
\hline Camada $(\mathrm{cm})$ & Coef. linear & Coef. angular \\
\hline 0 a 30 & 0,0029 & 0,5735 \\
30 a 270 & $-0,0035$ & 0,5811 \\
\hline
\end{tabular}



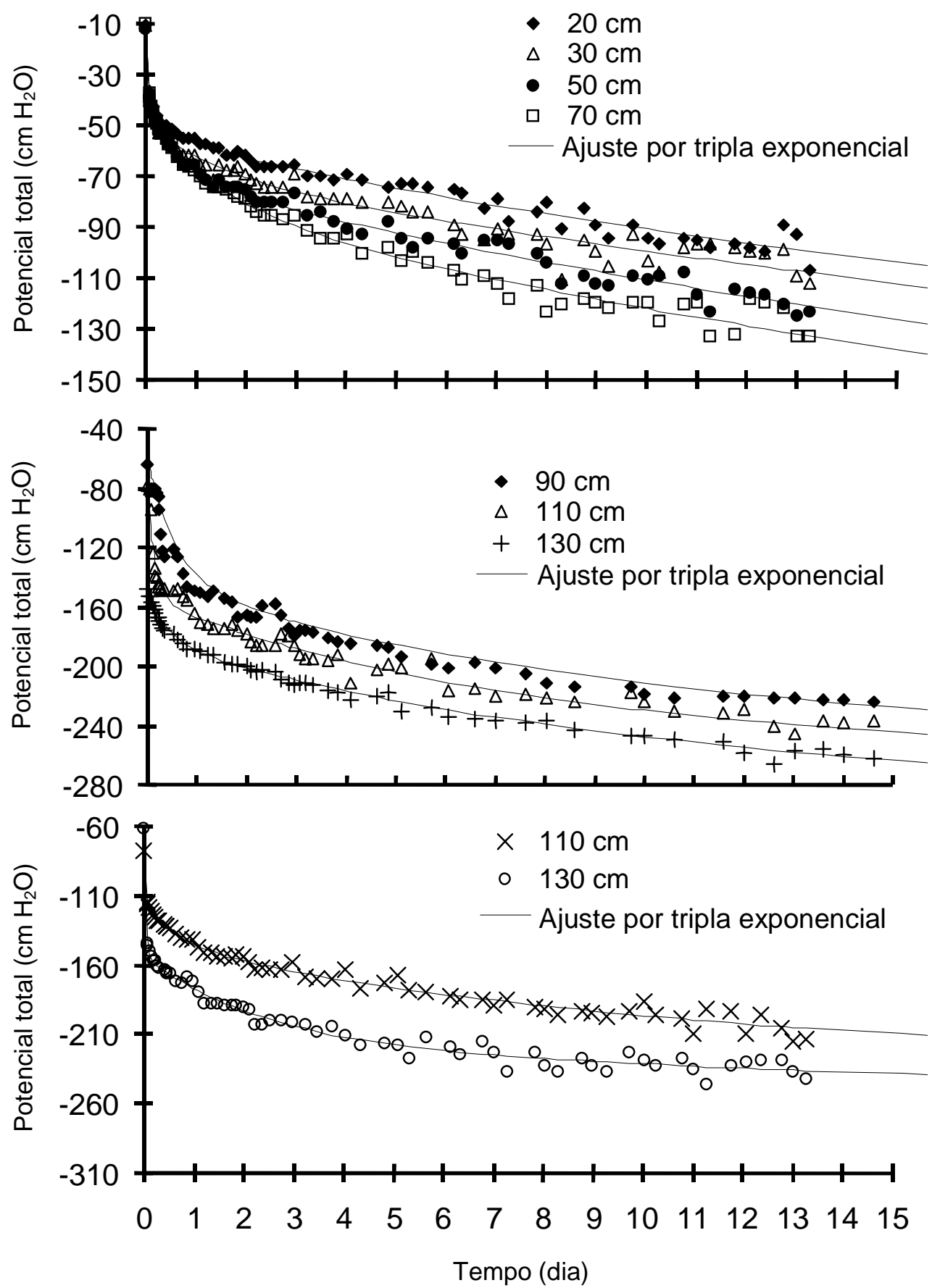

FIG. 1. Evolução dos potenciais totais da água no solo e curvas ajustada por uma tripla exponencial, para os experimentos 1 e 2 , em diferentes profundidades. 
todos os experimentos, pode-se verificar que os gradientes de potencial se estabilizam em valores aproximadamente constantes após a fase de drenagem rápida. $\mathrm{Na}$ Tabela 3 , são mostrados os coeficientes de determinação e os valores dos parâmetros da função analítica, usada para ajustar os potenciais totais da água do solo.

O estoque acumulado de água, relativo à espessura 0-25 cm (horizonte A), comporta-se de maneira comparável à umidade a $25 \mathrm{~cm}$, com uma fase de drenagem bastante rápida, passando abruptamente para a fase de drenagem lenta (Fig. 2). Nos horizontes mais profundos, ao contrário, o efeito de somatório traduz, como previsível, um comportamento mais uniforme: os horizontes BA e Bt se comportam de uma maneira comparável, com uma passagem bem marcada entre a fase rápida e a lenta, mais suave e atrasada em relação ao horizonte superficial. $\mathrm{Na}$ Tabela 4, são apresentados os parâmetros da função analítica, tripla exponencial, usada para o ajuste da evolução do estoque de água com o tempo, em várias profundidades, com os respectivos valores do coeficiente de determinação $\left(\mathrm{r}^{2}\right)$.
A representação dos resultados obtidos, em um sistema de coordenadas semilogarítmicas (Fig. 3), mostrou que as três relações $K(\theta)$ são praticamente lineares; isso significa que a forma das relações $\mathrm{K}(\theta)$ é próxima de uma exponencial. Uma relação $K(\theta)$ de forma exponencial é bastante comum na literatura. Vale a pena lembrar que a forma de $K(\theta)$ do tipo exponencial é geralmente conseqüência da escolha de um ajuste de forma logarítmica para a evolução cronológica das umidades e dos estoques acumulados de água (Vauclin \& Vachaud, 1987). Contudo, no presente caso não foi formulada, a priori, essa hipótese.

O horizonte A apresentou os valores de condutividade hidráulica mais elevados, atingindo, na faixa superior das umidades, valores entre $1.000 \mathrm{e}$ $10.000 \mathrm{~mm} \mathrm{dia}^{-1}$, compatíveis com valores de $100 \mathrm{~mm} \mathrm{~h}^{-1}$ de permeabilidade saturada medida pelo método do duplo anel, freqüentemente reportados para solos comparáveis. $\mathrm{O}$ horizonte $\mathrm{BA}$ apresentou valores menores na faixa de duas ordens de grandeza. Enfim, o horizonte $\mathrm{Bt}$ apresentou valores de aproximadamente uma ordem de grandeza menores em relação ao horizonte BA

TABELA 3. Parâmetros da função $\Psi=\mathbf{A}\left(\mathrm{e}^{-(\mathrm{Bt})}-1\right)+\mathbf{C}\left(\mathrm{e}^{-(\mathrm{Dt})}-1\right)+\mathbf{E}\left(\mathrm{e}^{-(\mathrm{Ft})}-1\right)+\mathbf{G}$ resultante dos ajustes da evolução do potencial total com o tempo, em várias profundidades, com os respectivos valores do coeficiente de determinação $\left(r^{2}\right)$ para os experimentos 1 e 2 .

\begin{tabular}{|c|c|c|c|c|c|c|c|c|}
\hline \multirow{2}{*}{$\begin{array}{l}\text { Profundidade } \\
\quad(\mathrm{cm})\end{array}$} & \multicolumn{7}{|c|}{ Parâmetros de ajuste } & \multirow{2}{*}{$\begin{array}{c}\mathrm{r}^{2} \\
(\%)\end{array}$} \\
\hline & $\mathrm{A}$ & $\mathrm{B}$ & $\mathrm{C}$ & $\mathrm{D}$ & $\mathrm{E}$ & $\mathrm{F}$ & $\mathrm{G}$ & \\
\hline & \multicolumn{8}{|c|}{ Experimento 1} \\
\hline 20 & $-31,9641$ & 17,21 & $-11,7213$ & 1,5752 & $-100,167$ & 0,04448 & $-11,1$ & 98,7 \\
\hline 30 & $-38,4389$ & 13,026 & $-19,3979$ & 0,65335 & $-140,653$ & 0,02455 & $-11,1$ & 98,5 \\
\hline 50 & $-30,614$ & 18,158 & $-27,4161$ & 1,2362 & $-135,277$ & 0,03543 & $-12,4$ & 99,2 \\
\hline 70 & $-15,18323$ & 40,4339 & $-54,9588$ & 1,2596 & $-115,094$ & 0,046169 & $-25,8$ & 99,1 \\
\hline 90 & $-14,2556$ & 293,406 & $-96,8206$ & 1,28008 & $-112,576$ & 0,03857 & $-14,9$ & 73,4 \\
\hline 110 & $-88,21101$ & 21,0639 & $-63,8573$ & 0,26835 & $-344,739$ & 0,034 & $-51,0$ & 98,5 \\
\hline \multirow[t]{2}{*}{130} & $-92,1849$ & 22,323 & $-65,2368$ & 0,4047 & $-66,3$ & 0,02291 & $-61,5$ & 98,6 \\
\hline & \multicolumn{8}{|c|}{ Experimento 2} \\
\hline 90 & -874769 & 0,13594 & $-75,0815$ & 1,6153 & $-343,179$ & 0,00269 & $-64,4$ & 99,3 \\
\hline 110 & $-82,3908$ & 0,15551 & $-76,1104$ & 6,6214 & $-476,353$ & 0,00221 & $-78,2$ & 98,5 \\
\hline 130 & $-73,8676$ & 0,12753 & $-34,6872$ & 2,6603 & $-435,697$ & 0,002871 & $-147,5$ & 99,6 \\
\hline
\end{tabular}



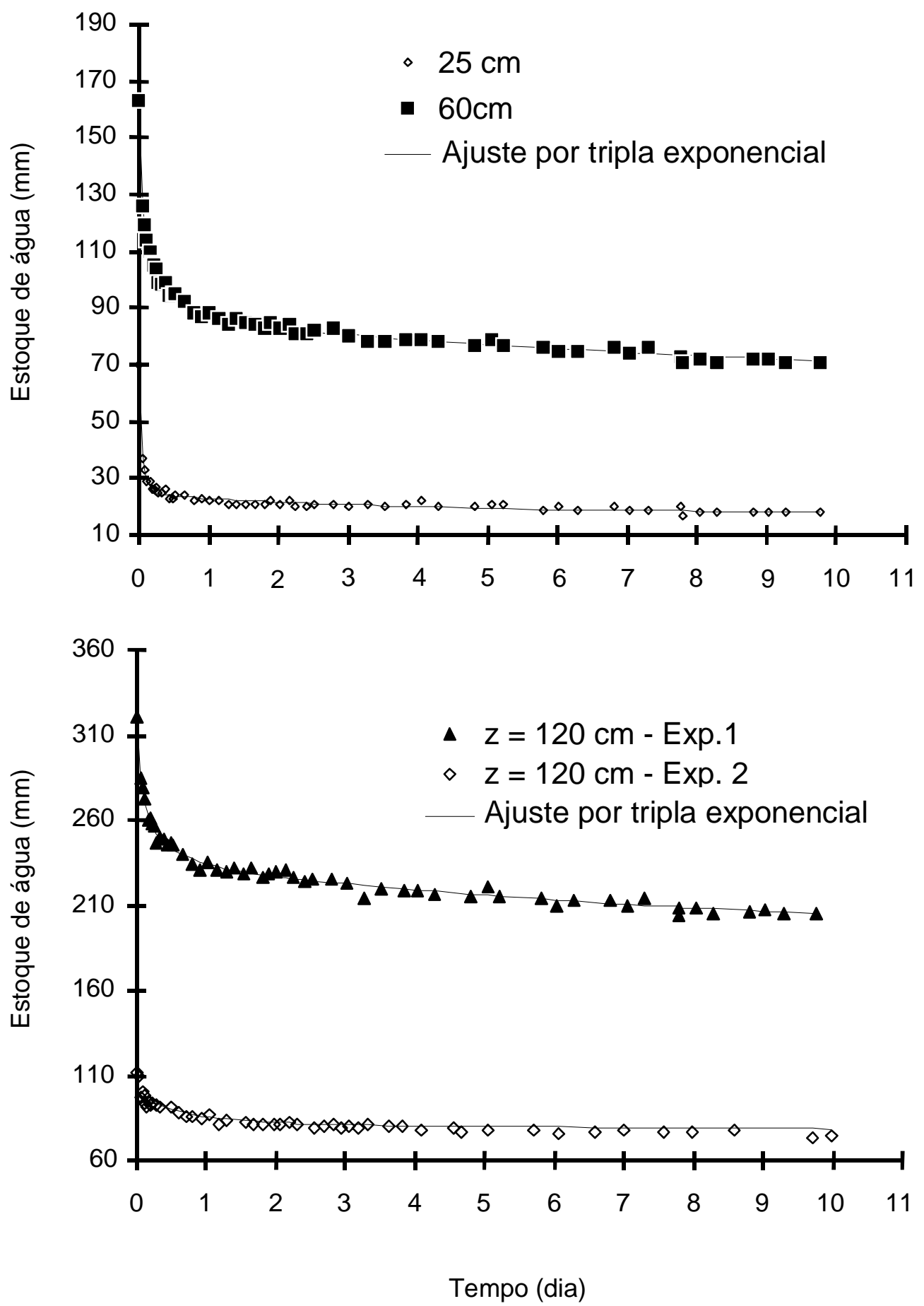

FIG. 2. Evolução do estoque de água no solo e curvas ajustada por uma tripla exponencial, para os experimentos 1 e 2 , em diferentes profundidades. 
Comparando esses resultados com os obtidos por Moreira \& Carneiro (1987), em Podzólico Amarelo localizado a $35 \mathrm{~m}$ do local deste estudo, constatouse uma boa concordância em relação aos horizontes A e BA, e uma discordância nítida quanto ao horizonte $\mathrm{Bt}$, que mostrou-se bem mais condutor. No perfil estudado por Moreira \& Carneiro (1987), não foi considerada a fase inicial de drenagem rápida. Assim, considerando tal fato como a causa provável daquela discordância, aplicou-se essa mesma metodologia após eliminação dos dados da fase de drenagem rápida do experimento. Os valores de $\mathrm{K}(\theta)$, assim obtidos, passaram a ser mais elevados na profundidade de $120 \mathrm{~cm}$, permanecendo, contudo, abaixo dos valores de $\mathrm{K}(\theta)$ para $60 \mathrm{~cm}$. Portanto, mesmo que existam diferenças atribuíveis à metodologia, a sua influência não foi suficiente para inverter, em relação à profundidade, a seqüência observada neste trabalho. Por sua vez, esse teste permitiu constatar que $\mathrm{o}$ ajuste por tripla exponencial foi melhor do que o ajuste logarítmico, sobretudo, por apresentar uma repartição mais aleatória dos resíduos.

Os valores obtidos de $K(\theta)$ são afetados pelos erros inerentes à determinação das densidades de fluxo e dos gradientes de potencial, obtidos com base nos ajustes das umidades e dos potenciais totais. No caso de ajustes manuais, não é possível avaliar o erro final, sendo extremamente complicado fazer um cálculo de propagação de erros, no caso dos ajustes analíticos, uma vez que a umidade é afetada pelos erros de ajuste.

Ajustar os dados na zona de transição entre a fase rápida e a fase lenta, sempre foi difícil tanto pelo método manual como analítico, seja no que concerne à sua localização, seja no que se refere ao seu raio de curvatura. Além disso, quando se pretende reproduzir esse comportamento, algumas formas analíticas, encontradas na literatura, não podem ser aplicadas. Com efeito, as formas suscetíveis de reproduzir a zona de transição são: a função potência e a soma de duas ou, melhor ainda, três exponenciais. Deve-se observar que, além de reproduzir de modo adequado a zona de transição, tal ajuste deve fornecer um comportamento consistente com o conjunto inteiro dos dados experimentais.

No que diz respeito à precisão dos valores de condutividade hidráulica, podem ser destacadas as seguintes considerações: 1) a precisão na zona de transição é menor do que para os trechos de drenagem rápida e lenta que a enquadram, e parte da dispersão dos dados de $\mathrm{K}(\theta)$, nesta região, pode ser atribuída às limitações inerentes à qualidade dos dados disponíveis e à metodologia utilizada; 2) um erro de $\pm 50 \%$ sobre o gradiente de potencial provocará um valor de condutividade hidráulica final multiplicado ou dividido por 2, ao passo que um erro na curvatura no estoque $(\mathrm{W}(\mathrm{t}))$ pode induzir uma ou mais ordens de grandeza no valor final da condutividade hidráulica .

A Fig. 4 mostra a condutividade hidráulica no horizonte $\mathrm{Bt}$ antes e depois de retirada uma camada de $80 \mathrm{~cm}$ de solo que inclui os horizontes A e BA.

O aumento de $K(\theta)$ pode ser causado pela grande variabilidade do horizonte $\mathrm{Bt}$, conseqüência da heterogeneidade do sedimento original, associado a uma diminuição do teor de argila com a profundidade (Brasil, 1969).

TABELA 4. Parâmetros da função $\mathbf{W}=\mathbf{A}\left(\mathrm{e}^{-(\mathrm{Bt})}-1\right)+\mathrm{C}\left(\mathrm{e}^{-(\mathrm{Dt})}-1\right)+\mathbf{E}\left(\mathrm{e}^{-(\mathrm{Ft})}-1\right)+\mathbf{G}$ resultante dos ajustes da evolução do estoque de água com o tempo, em várias profundidades, com os respectivos valores do coeficiente de determinação $\left(r^{2}\right)$ para os experimentos 1 e 2 .

\begin{tabular}{|c|c|c|c|c|c|c|c|c|}
\hline \multirow{2}{*}{$\begin{array}{l}\text { Profundidade } \\
\text { (cm) }\end{array}$} & \multicolumn{7}{|c|}{ Parâmetros de ajuste } & \multirow{2}{*}{$\begin{array}{l}\mathrm{r}^{2} \\
(\%)\end{array}$} \\
\hline & $\mathrm{A}$ & $\mathrm{B}$ & $\mathrm{C}$ & $\mathrm{D}$ & $\mathrm{E}$ & $\mathrm{F}$ & $\mathrm{G}$ & \\
\hline & \multicolumn{8}{|c|}{ Experimento 1} \\
\hline 25 & 45,2787 & 18,515 & 130,387 & 0,00050 & 6,29628 & 0,31357 & 70 & 97,81 \\
\hline 60 & 27,2803 & 2,4674 & 23,3482 & 0,11908 & 48,1272 & 17,312 & 163 & 99,43 \\
\hline \multirow[t]{2}{*}{120} & 29,5634 & 2,1614 & 45,3816 & 0,11443 & 55,7698 & 12,612 & 321 & 98,97 \\
\hline & \multicolumn{8}{|c|}{ Experimento 2} \\
\hline 120 & 8,02631 & 0,06416 & 16,27748 & 1,40434 & 10,83197 & 1109,5 & 109,443 & 97,5 \\
\hline
\end{tabular}




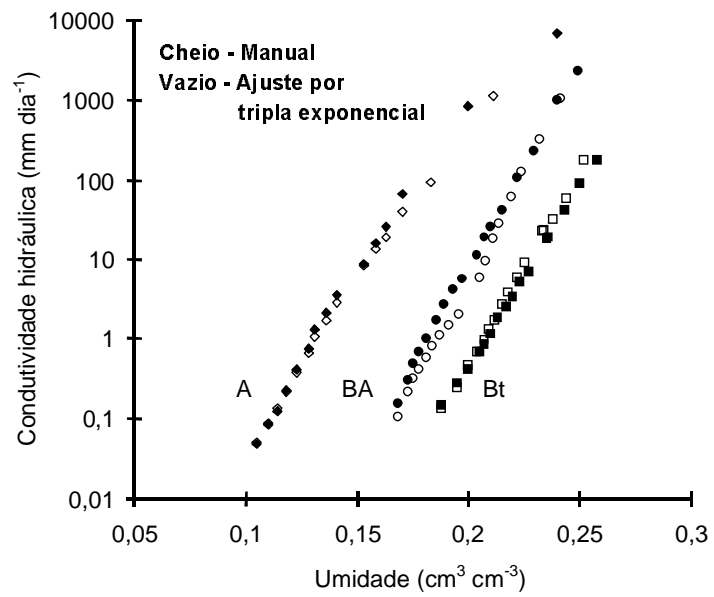

FIG. 3. Condutividade hidráulica como função da umidade, nos horizontes A, BA e Bt (Experimento 1).

\section{CONCLUSÕES}

1. Entre as funções testadas, só os tipos potência, soma de duas exponenciais e soma de três exponenciais têm capacidade de reproduzir adequadamente a transição entre a fase rápida e a fase lenta da drenagem.

2. O horizonte BA, de estrutura maciça, coesa e com poucos poros visíveis, é um horizonte de impedimento para a infiltração da água.

3. Não há aumento da condutividade hidráulica no horizonte $\mathrm{Bt}$.

\section{REFERÊNCIAS}

BRASIL. Ministério da Agricultura. Departamento Nacional de Pesquisa Agropecuária. Divisão de Pesquisa Pedológica. Levantamento exploratório-reconhecimento de solos do Estado de Pernambuco. Recife, 1972. 2v. (DNPEA. Boletim Técnico, 26; SUDENEDRN. Série Pedologia, 14).

BRASIL. Ministério da Agricultura. Escritório de Pesquisas e Experimentação. Equipe de Pedologia e Fertilidade do Solo. Levantamento detalhado dos solos da Estação Experimental de Itapirema. Rio de Janeiro, 1969. 84p. (Boletim Técnico, 12).

CHONG, S.K.; GREEN, R.E.; AHUJA, L.R. Simple in situ determination of hydraulic conductivity by power function description of drainage. Water Resources Research, Washington, v.17, p.1109-1114, 1981.

HILLEL, D.; KRENTOS, V.D.; STYLLIANOU, Y Procedure and test of an internal drainage method for

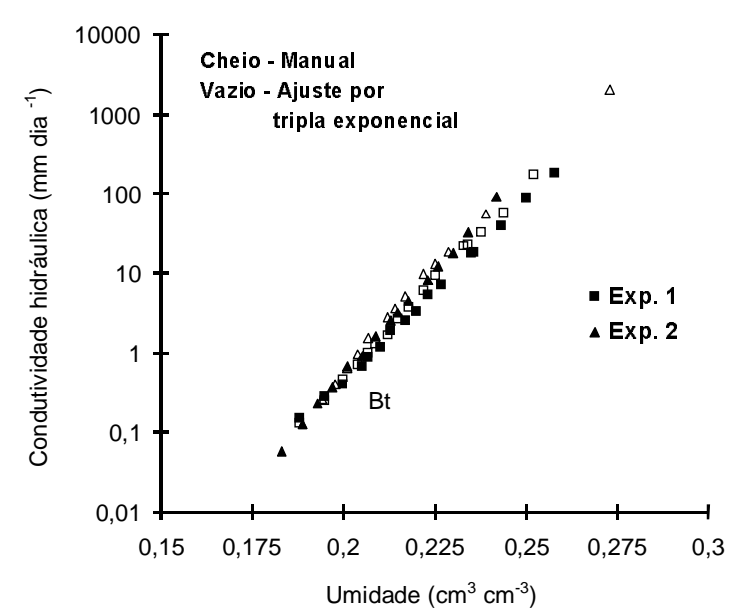

FIG. 4. Condutividade hidráulica como função da umidade, nos experimentos 1 e 2, no horizonte Bt.

measuring hydraulic characteristics in situ. Soil Science, Baltimore, v.144, p.395-400, 1972

LIBARDI, P.L.; REICHARDT, K.; NILSEN, D.R.; BIGGAR, J.W. Simple field methods for estimating soil hydraulic conductivity. Soil Science Society of America. Journal, Madison, v.44, p.3-7, 1980.

MOREIRA J.A.A.; CARNEIRO C.J.G. Características de retenção de água de um solo Podzólico VermelhoAmarelo. Pesquisa Agropecuária Brasileira, Brasília, v.22, n.4, p.411-418, abr. 1987.

PREVEDELLO, B.M.S.; PREVEDELLO, C.L.; LIBARDI, P.L. Simplificação analítica do método do perfil instantâneo para obtenção da condutividade hidráulica não saturada em condições de campo. Revista Brasileira de Ciência do Solo, Campinas, v.5, p.93-97, 1981

SAUNDERS, L.C.U. Métodos de determinação e variabilidade espacial da condutividade hidráulica sob condições de campo. Piracicaba : ESALQ, 1978. 71p. Tese de Doutorado.

VACHAUD, G.; VAUCLIN, M.; LATY, R. Caractérisation hydrodinamique in situ de quelques sols SoudanoSahéliens à l'aide d'un programme de calcule assité par micro-ordinateur. In: SIVAKUMAR, M.V.K.; WALLACE, J.S.; RENARD, C.; GIROUX, C. (Ed.) Soil water balance in the Sudano-Sahelian zone Niamey : International Association of Hydrologic Sciences, 1991. p.175-185.

VAUCLIN, M.; VACHAUD, G. Caractérisation hydrodinamique des sols: analyse simplifiée des essais de drainage interne. Agronomie, Paris, v.7, n.9, p.647$655,1987$. 